\title{
Direct access to investigational interventions outside the trial process: ethical reflections on the TOTAL-trial debate
}

\author{
N. Crombag ${ }^{1 *}$, D. Pizzolato ${ }^{2 *}$, K. Dierickx ${ }^{2}$
}

${ }^{1}$ KU Leuven, Department Development and Regeneration, B-3000, Leuven Belgium.

${ }^{2}$ KU Leuven, Department of Public Health and Primary Care, Centre for Biomedical Ethics and Law, B-3000, Leuven Belgium.

\section{Corresponding author:}

Neeltje Crombag

KU Leuven,

Department Development and Regeneration, B-3000,

Leuven Belgium

neeltje.crombag@kuleuven.be

*both authors contributed equally

Short title: Ethical reflections on the TOTAL-trial debate

Key words: Randomized controlled trials, Congenital diaphragmatic hernia, Fetoscopic endoluminal tracheal occlusion, Off-trial access, Expanded trial access, Right to Try been through the copyediting, typesetting, pagination and proofreading process which may lead to differences between this version and the Version of Record. Please cite this article as doi: 10.1002/uog.23132 
In severely ill patients, when a priori chances of survival are low, a promising investigational intervention can be seen as the last hope, or the last straw, to improve the patient's chances of survival. At the same time, this might interfere with the ethical principles on which research and clinical care are based.

Direct access to the investigational intervention outside the trial process, for fetuses with severe (left-sided) congenital diaphragmatic hernia (CDH), has recently been debated at the 18th World Congress in Fetal Medicine ${ }^{1}$. Whether to expand the access of the investigational intervention outside the trial process, or to keep the experimentation confined within the clinical research until the end of the approval process were the two contrasting opinions debated. The discussants debated whether FETO should be offered exclusively within an RCT or also offered outside the research setting. Here we reflect on both opinions published in Ultrasound in Obstetrics and Gynecology 2,3 .

This commentary summarizes some of the ethical issues derived from this debate: the randomization process, autonomy in decision-making/ concept of responsibility in clinical research, beneficence/ non-maleficence and the individual/ public interest (Table 1). By elaborating on these issues, this paper aims at providing an ethical reflection on this debate. While the debate and the contrasting opinions regarding the TOTAL-trial here serve as our key example, this reflection is relevant for any RCT involving fetal medicine. The relatively rareness and detection rates of the conditions, as 
well as the generic challenges of patient recruitment in fetal treatment trials complicate running RCTs in fetal medicine.

Well-designed RCTs are based on the principle of equipoise: a genuine uncertainty regarding benefits and risks of an investigational intervention as compared to the current best-proven therapy within the scientific community ${ }^{3,45}$ In these circumstances, harms and benefits of participation in a trial are balanced, and therefore allocation to either arm is considered ethical. In clinical research, randomization is the 'golden standard'. However, in specific conditions, the randomization process may be seen as extremely unfair, violating the principle of justice ${ }^{6}$. In RCTs, in particular those involving the development of life-saving treatments, the unfairness of the randomization process may be seen as prohibiting direct access to potential benefits of a new intervention ${ }^{7,8}$. In the case of a severely ill patient, or this case, of an unborn child with an a priori low chance of postnatal survival, randomization for a trial potentially elaborates dilemmas among physicians-researchers. On the one hand, under the Hippocratic Oath physicians are committed to providing the best available treatment possible, while on the other hand, as a researcher, they are obliged to work according to the scientific rules, to provide the highest level of evidence for the best-proven therapy ${ }^{9}$. Randomly allocating a patient to either the standard therapy with its known a priori low chance of survival or, alternatively, to a relatively new intervention potentially increasing survival, might interfere with the physicians’ professional commitments. The principle of beneficence ${ }^{6}$, the intent of doing good for the patient involved, might direct physicians towards offering investigational (potential) life-saving interventions outside the trial process, as 
a mean to take advantage of all benefits the new potential therapy might have, without looking at potential harm. 
RCTs are designed according to predefined protocols, in order to provide scientifically sound evidence, as well as guaranteeing the ethical character of randomization ${ }^{9}$. By prohibiting direct access to an investigational intervention, participants are protected from unneeded harm. This intent of avoiding harm can be seen as the principle of nonmaleficence $^{6}$, and also applies to severely ill research participants.

Providing the option to ask directly for the experimental intervention allows people with life-threatening conditions to appeal upon their full decisional autonomy, demanding access to all possible options ${ }^{10}$. Depriving severely-ill people from asking for the investigational intervention can be seen as a restriction of this autonomy. However, limiting the access to experimental interventions of which benefits and risks are still unclear, is also a protective measure. Besides, clinicians/researchers have a responsibility to execute the research in line with the study design protocol and scientific rules, in order to provide the best evidence possible for future patients. This concept of responsibility in clinical research, linked with the principle of nonmaleficence, allows clinicians/researchers to act as a gatekeeper, avoiding unintentional and unnecessary harms and risks for research participants.

For patients to request direct access to an investigational intervention can be seen as the Right To Try (RTT). The RTT originates from USA legislation and allows terminally ill patients to request access to investigational therapies outside the experiment ${ }^{11}$. RTT implies the patient's right to preserve life when there is the hope that an intervention might be successful, meaning that in specific situations patients have the possibility to try an investigational intervention, under their own responsibility ${ }^{11}$. Relying on the 
possibility to be allowed to use risky interventions can be viewed as a sort of right, based on the conceptual framework of personal autonomy and freedom of choice ${ }^{12}$. RTT is based on people's self-interest in taking all actions that might be needed to have a last option. However, RTT also has downsides. It can create the so-called "illusion of access”, giving patients the perception that they can ask for any possible investigational interventions ${ }^{9}$, while restrictions in research are put in place for protecting research participants. Moreover, from a public interest perspective, giving access to experimental interventions delays the approval process prolonging the time for finding the needed evidence $^{13}$. While providing direct access to off-trial experimental interventions may favor the individual interest, it negatively impacts the public interest. Providing investigational interventions outside a research setting interferes with the process of evidence finding, hence undermining rapid access to new therapies for future patients. Besides, allowing off-trial access might cause liability-related issues as experimental interventions might cause unintentional, unpredictable and serious harm. In the case of a severe and rare disease, finding the best-proven therapy is even more challenging. Because of the low number of patients affected by a rare condition, these trials challenge the inclusion process, consequently prolonging the trial duration. The duration of the trial demands patience but might cause trial fatigue, potentially hindering finding the highest level of evidence ${ }^{14}$. Ideally, new interventions should only become accessible, after providing evidence on effectivity and safety, in order to maintain the scientific integrity of clinical trials 9 . 
This article is protected by copyright. All rights reserved. 
Clinical research and clinical practice are two distinct entities. In proposing or not an investigational treatment outside of RCTs, the main clash is rooted in the conflict between the patient's autonomy of having the right to make their own decisions and the concept of responsibility in clinical research to protect research participants for making imprudent decisions. Moreover, it is balancing the goals of clinical care, the individual patient's interest with the goals of research, the public interest. In daily practice, this means as a physician to provide the care in the best interest of the patient versus as a researcher the obligation to protect research integrity for finding the best available evidence for future patients ${ }^{15}$. 


\section{References}

1. The Fetal MedicineFoundation. Final programme 18th World Congress in Fetal Medicine. Available from:

https://fetalmedicine.org/var/uploads/18thWCFMprogram.pdf [August 20th 2020].

2. Ville Y. Should we offer fetal surgery for severe congenital diaphragmatic hernia or bring those cases to trial? The difference between chance and hazard. Ultrasound Obstet Gynecol 2020. DOI: 10.1002/uog.22103.

3. Deprest J. Prenatal treatment of severe congenital diaphragmatic hernia: there is still medical equipoise. Ultrasound Obstet Gynecol 2020. DOI:

10.1002/uog.22182.

4. Guyatt GH, Haynes RB, Jaeschke RZ, Cook DJ, Green L, Naylor CD, Wilson MC, Scott RW. Users' guides to the medical literature: XXV. Evidence-based medicine: Principles for applying the users' guides to patient care. JAMA 2000; 284(10):1290-6.

5. Sibbald B, Roland M. Understanding controlled trials: Why are randomised controlled trials important? BMJ 1998; 316(7126):201.

6. Freedman B. Equipoise and the Ethics of Clinical Research. N Engl J Med 1987; 317(3):141-5.

7. Beauchamp TL, Childress JF. Principles of Biomedical Ethics. 7th ed. University Press: Oxford, 2012. 
8. Colli A, Pagliaro L, Duca P. The ethical problem of randomization. Intern Emerg Med 2014; 9:799-804.

9. Neuhaus CP, Zacharias RL. Compassionate use of gene therapies in pediatrics: An ethical analysis. Semin Perinatol 2018; 42(8):508-514.

10. Joffe S, Miller FG. Bench to Bedside: Mapping the Moral Terrain of Clinical Research. Hastings Cent Rep 2008; 38(2):30-42.

11. Varelius J. The value of autonomy in medical ethics. Med Health Care Philos 2006; 9(3):377-88.

12. Carrieri D, Peccatori FA, Boniolo G. The ethical plausibility of the 'Right To Try' laws. Crit Rev Oncol Hematol 2018; 122:64-71.

13. Riva L, Campanozzi L, Vitali M, Ricci G, Tambone V. Unproven stem cell therapies: is it my right to try? Ann Ist Super Sanità 2019; 55(2):179-185.

14. Okie S. Access before approval - A right to take experimental drugs? N Engl J Med 2006; 355(5):437-40.

15. Basurto D, Russo FM, Van der Veeken L, Van der Merwe J, Hooper S, Benachi A, De Bie F, Gomez O, Deprest J. Prenatal diagnosis and management of congenital diaphragmatic hernia. Best Pract Res Clin Obstet Gynaecol 2019;58: 93-106.

16. Miller FG, Rosenstein DL, DeRenzo EG. Professional integrity in clinical research. JAMA 1998; 280(16):1449-54. 
Expanded trial access

Unfairness of randomization

Beneficence

Autonomous decision-making

life/ death situations

\section{FETO exclusively within RCT}

Randomization as the golden standard

Non-maleficence

Concept of responsibility in clinical

research

Obligation to provide intervention with the highest level of evidence

Tabel 1 Ethical issues originated from the debate 'Severe CDH: debate on FETO outside the TOTAL trial' 
